\title{
Determination of the Optical Properties of Carbonaceous Aerosols by Monochromated Electron Energy-loss Spectroscopy
}

\author{
Jiangtao Zhu, Peter A. Crozier and James R. Anderson
}

School for Engineering of Matter, Transport and Energy, Arizona State University, Tempe, AZ, USA, 85287

Carbonaceous aerosols, primarily from anthropogenic combustion sources, significantly affect global radiative forcing. It is essential to determine their optical properties to understand their contributions to climate change. Conventional light-optical technologies usually measure a bulk mixture of aerosol types and can supply information only in a limited spectral range. Electron energy-loss spectroscopy (EELS) is a promising way to derive the dielectric functions over the full spectral range on individual aerosol particle [1]. We have analyzed the size distribution, morphology, structure and chemical composition of aerosols collected from East Asia by transmission electron microscopy (TEM) and found in these samples there are three main classes of carbonaceous aerosols: brown carbon spheres, large spherule soot and small spherule soot [2]. Here we show their different optical properties from different types of carbonaceous aerosols by EELS. We also found their differences in refractive indices are directly related to their $\mathrm{sp}^{2} /\left(\mathrm{sp}^{2}+\mathrm{sp}^{3}\right)$ ratio.

There are several points that must be considered for the EELS collection and analysis related to atmospheric aerosols. The specimens collected from the atmosphere contain different types of carbonaceous aerosols some of which are electron beam sensitive especially under scanning TEM mode. Brown carbon sphere aerosols are beam sensitive and we collected EELS from them in TEM image mode from the center of the spheres. For soot, which is resistant to beam damage, STEM mode was used to collect the spectra after first baking the sample at $150^{\circ} \mathrm{C}$ in vacuum to reduce contamination. All the low-loss EELS were collected on the monochromated microscope (TEAM 1) at National Center of Electron Microscopy with energy resolution of $0.15-0.2 \mathrm{eV}$ at $80 \mathrm{kV}$.

As shown in Fig. 1a, the amorphous brown carbon spheres are normally isolated with diameter larger than $100 \mathrm{~nm}$. Large spherule soot consists of aggregates composed of graphitic spherules with diameters as large as $100 \mathrm{~nm}$ (Fig.1b). Small spherule soot is composed of tens of graphitic spheres with diameters of about $30 \mathrm{~nm}$ (Fig.1c). Their single scattering distributions (Fig. 2a) derived from the EELS show clearly their difference especially below $10 \mathrm{eV}$ which is in the UV, visible and infrared range.

For the optical properties determination, the thin film approximation as discussed in these proceedings [3]. Kramers-Kronig analysis was applied to derive dielectric functions with the normalization of the energy-loss function by the thickness. The derived refractive indices in the range of 200-1200 $\mathrm{nm}$ (1.03-6.2 eV) of the three types of carbonaceous aerosols show quite different optical properties (Fig. 2b and c). All of them are wavelength dependent. Both large and small spherule soot have a higher absorption ability (higher k value) than that of brown carbon spheres. It is found that there is a variance of optical properties even within one class of carbonaceous aerosols. As shown in Fig. $2 d$, we found that both $\mathrm{n}_{550 \mathrm{~nm}}$ and $\mathrm{k}_{550 \mathrm{~nm}}$ of these carbonaceous materials increase with their ratio of $\mathrm{sp}^{2} /\left(\mathrm{sp}^{2}+\mathrm{sp}^{3}\right)$. Further study will include finding an improved method to remove the surface and interface contributions for the small spherule soot aggregate, detecting the $\mathrm{C}-\mathrm{H}, \mathrm{C}-\mathrm{O}$ 
vibration modes in the aerosols and deriving their optical properties in the infrared range using the monochromated NION STEM 100 currently installed at Arizona State University [4].

\section{References}

[1] D.T.L. Alexander, P.A. Crozier, J.R. Anderson, Science 321 (2008), p. 833.

[2] J. Zhu, P.A. Crozier, J.R. Anderson, Atmos Chem Phys Discuss. 12 (2012), p. 32945.

[3] J. Zhu, P.A. Crozier, J.R. Anderson, ibid, these proceedings.

[4] The authors acknowledge support of the National Center for Electron Microscopy, and the National Institute of Standards and Technology under Award 60NANB10D022. The authors acknowledge John M.Cowley Center for High Resolution Microscopy at Arizona State University.
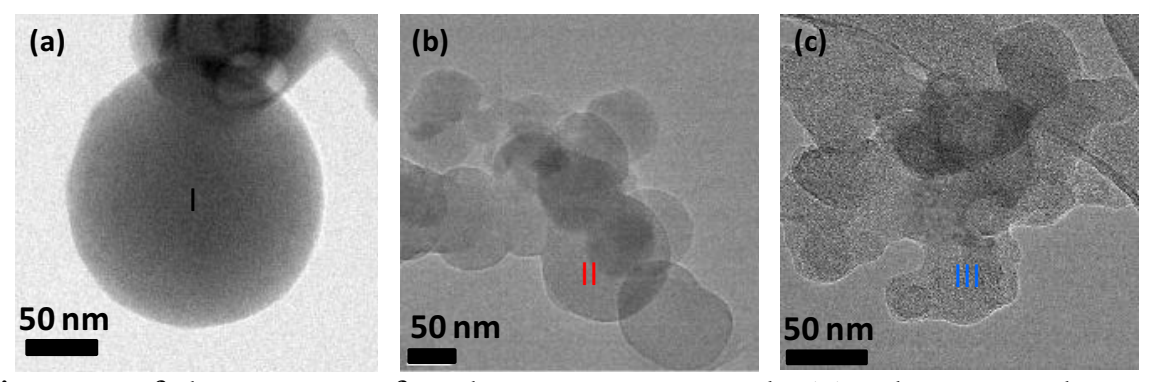

Figure 1. TEM images of three types of carbonaceous aerosols (a) a brown carbon sphere, (b) large spherule graphitic soot, and (c) small spherule graphitic soot.
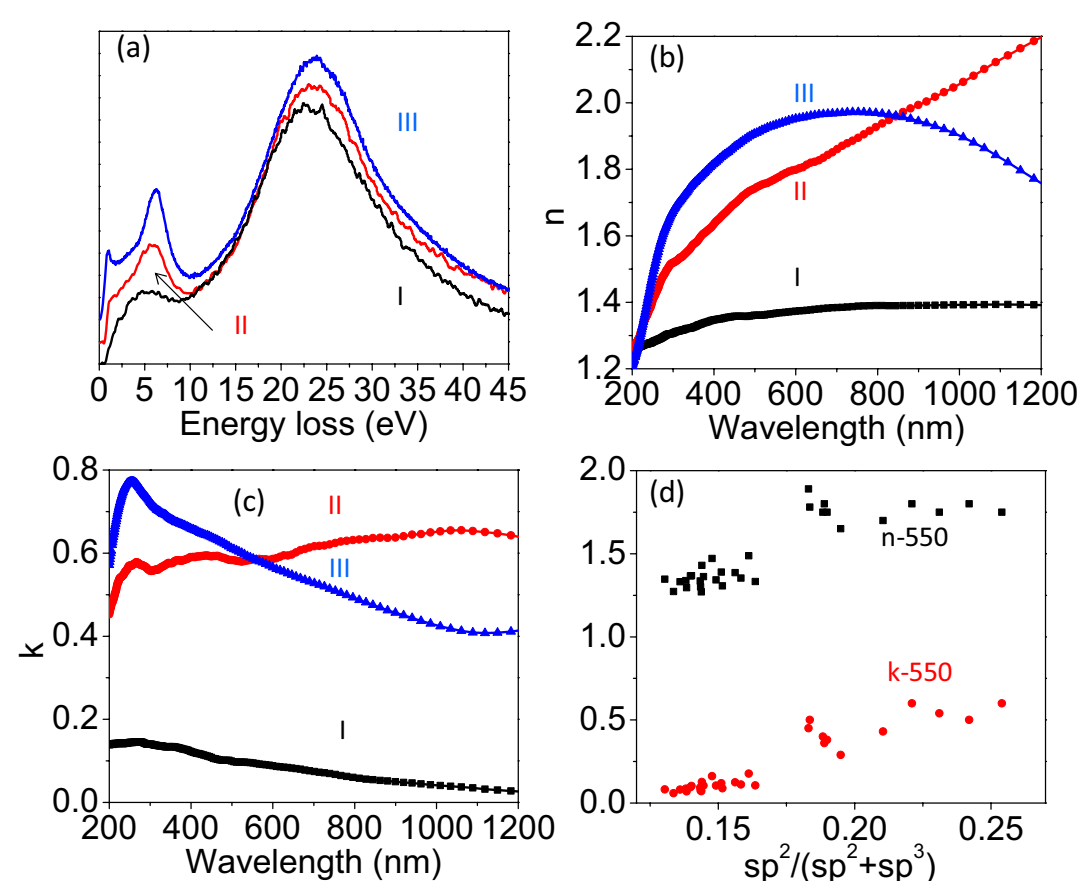

Figure 2. (a) Single scattering distribution of brown carbon sphere (I, in black), large spherule soot (II, in red) and small spherule soot (III, in blue). Real and imaginary part of refractive indices (n, k) of the three aerosols derived in wavelength of 200-1200 nm are shown in (b, c), respectively. (d) The relation of the refractive indices at wavelength of $550 \mathrm{~nm}$ of brown carbon spheres, and large soot spherules with their $\mathrm{sp}^{2} /\left(\mathrm{sp}^{2}+\mathrm{sp}^{3}\right)$ ratio. 